\title{
Lumbar Spinal Stenosis: Ipsilateral Facet-sparing Unilateral Laminotomy for Bilateral Decompression: Technical Note and Preliminary Results
}

\author{
Luca Papavero*, Kathrin Schawjinski*, Nawar Ali, Justus Oehm, Markus Pietrek, Karsten Schoeller \\ Clinic for Spine Surgery, Schoen Clinic Hamburg Eilbek, Hamburg, Germany
}

Received: September 24, 2021

Revised: October 2, 2021

Accepted: October 16, 2021

Corresponding Author:

Luca Papavero, MD, PhD

Clinic for Spine Surgery, Schoen

Clinic Hamburg Eilbek, Dehnhaide

120, D-22081 Hamburg, Germany

Tel: +49-40-20927018

Fax: +49-40-20927005

E-mail: Luca.Papavero@yahoo.de

*These authors contributed equally to the work and share the first authorship.

\begin{abstract}
Microsurgical unilateral laminotomy for bilateral decompression (ULBD) decompresses effectively lumbar stenosis. Whenever low grade degenerative spondylolisthesis, sagittal oriented facet joints, scoliotic deformity, tall disc, and obesity jeopardize postoperative segmental stability, a maximum facet-preserving decompression is desirable. The medialized ULBD (mULBD) aims to preserve the facet joint on the approach side. Sixty-four patients presenting with neurogenic claudication underwent single or multilevel decompression with ULBD or mULBD according to the judgement of the surgeon. The volume of the target facet joints and the dural cross sectional area (CT-DCSA) were measured pre- and postoperatively by an ultra-low-dose CT with a specific software. Forty-three pairs of facet joints were addressed with ULBD and 43 pairs with mULBD. Postoperatively, the mean percentage of the preoperative facet joint volume preserved on the approach side was 70\% $\pm 4 \%$ (ULBD) and $88 \% \pm 6 \%(m U L B D) ;(p<0.001)$. The mean facet joint volume preserved contraleral to the approach side was $87 \% \pm 6 \%$ (ULBD) and $91 \%$ $\pm 6 \%$ (mULBD); $(p=0.4)$. The mean postoperative CT-DCSA was $152 \pm 30 \mathrm{~mm}^{2}$ (ULBD) and $153 \pm 26 \mathrm{~mm}^{2}$ ( $\left.\mathrm{mULBD}\right) ;(p=0.43)$. The mUBLD decompresses lumbar spinal stenosis effectively as ULBD and preserves better the facet joint on the approach side.
\end{abstract}

Key Words: Zygapophyseal joint, Spinal stenosis, Spinal canal, Decompression, Spondylolisthesis

\section{INTRODUCTION}

Microsurgical unilateral laminotomy for bilateral decompression (ULBD) provides an effective and largely stability preserving treatment for lumbar spinal stenosis (LSS) [1,2]. The advantages of the technique are the paramedian less muscle tissue traumatizing approach, either if a tubular retractor or a miniaturized speculum retractor is inserted, and the complete preservation of the dorsal midline inter- and supraspinous anatomical structures. However, a limitation is the larger facet joint (FJ) resection on the approach side compared with the contra- lateral one. Therefore, in multisegemental decompression the "slalom technique" has been recommended in order to distribute the biomechanical load on the weakened FJs [3].

The muscle-sparing interlaminar decompression (MILD) is also a minimally invasive surgical technique for LSS $[4,5]$. The strict midline approach weakens at some extent the ligaments and the adjacent spinous processes. However, it spares the muscle tissue and allows for an excellent funnel-shaped view of the intraspinal space. Furthermore, the FJ can be mostly preserved or, if needed, drilled off symmetrically.

This note describes a surgical technique which aims to com-

Copyright (C) 2021 Korean Minimally Invasive Spine Surgery Society

This is an Open Access article distributed under the terms of the Creative Commons Attribution Non-Commercial License (http://creativecommons.org/licenses/by-nc/4.0/) which permits unrestricted non-commercial use, distribution, and reproduction in any medium, provided the original work is properly cited. 
bine the advantageous paramedian approach of the ULBD sparing the midline ligaments with the facet-sparing decompression of the MILD procedure. For the sake of simplicity this blended technique is named the medialized ULBD (mULBD).

\section{MATERIALS AND METHODS}

\section{Patients and Methods}

The inclusion criteria were as follows: (1) Neurogenic claudication after failure of an adquate conservative therapy and with imaging confirming a single or multi-level LSS; (2) Minimum age: 75 years. This lower age limit was chosen with regard to the radiation protection; (3) Informed consent to undergo a pre/postoperative ultra-low-dose CT (ULD-CT) of the target facet joints. The exclusion criteria were: (1) previous surgery of the index level/s; (2) spondylolisthesis classified as Meyerding grade 2 or higher.

\section{Choice of Surgical Procedure}

Five board certified orthopedic or neurological surgeons (range of experience: from 6 to 30 years) performed the procedures. In a consensus conference it was agreed that the following parameters increase the potential risk of postoperative segmental instability: (1) disc height $>6.5 \mathrm{~mm}$; (2) mobile spondylolisthesis Meyerding grade 1; (3) degenerative scoliosis with Cobb angle $>15^{\circ}$; (4) facet joint effusions; (5) high sagittal orientation of the facet joints; (6) short interfacettal distance; and (7) $\mathrm{BMI}>30$. The presence of one or more of the mentioned factors led the surgeon to choose the mULBD technique. In planning a multi-level decompression the hybrid choice, i.e. ULBD at one level and mULBD at a different level, was also given.

\section{Surgical Technique}

Both microsurgical ULBD and MILD have been described in detail in the past $[1,2,5]$. The differences between ULBD and mULBD are summarized in Table 1 and shown in Figure 1. The following description refers to the use of a table fixed, miniaturized speculum-retractor but a tubular retractor is suitable as well (Figure 2).

In our hands mULBD is performed best with the help of a microscope from skin o skin. For safety reasons, the patient is secured with a belt on the gluetal area. This becomes helpful when the table has to be tilted. A 2-cm skin incision does not allow a "seek and find" surgery. The needle for preoperative labeling of the target level is inserted contralateral to the intended surgical side to avoid subcutaneous or intramuscular hematoma and 5-10 mm off the midline to prevent inadvertent cerebrospinal fluid leakage. The incised fascia is secured with few holding sutures. The paramedian muscle tissue is retracted from the interspinous ligament and from the lateral surface of the spinous process (SP). Any ipsilateral thickening of the tip of the SP is flattened with the diamond dust coated burr. A miniaturized speculum or an expandable tubular retractor is centered on the spinolaminar junction. The ipsilateral deep half of the SP is drilled off until the cranial insertion of the yellow ligament (YL) becomes visible. Furthermore, the basal SP should be thinned out contralaterally until the mid-

Table 1. Comparison of the key surgical steps between ULBD and mULBD

\begin{tabular}{|c|c|c|}
\hline & ULBD & $m U L B D$ \\
\hline Skin incision & Skin incision of $25 \mathrm{~mm}$ length - $10 \mathrm{~mm}$ paramedian & The same \\
\hline Soft tissue dissection & $\begin{array}{l}\text { Incision of subcutaneous tissue and fascia, blunt splitting of } \\
\text { paraspinous muscle tissue. }\end{array}$ & $\begin{array}{l}\text { The same and, if necessary, drill the prominent tip of the SP } \\
\text { with the diamond dust coated burr flat to its lateral sur- } \\
\text { face. }\end{array}$ \\
\hline Insertion of the retractor & $\begin{array}{l}\text { Center on the lamino-facet junction and on the inferior edge } \\
\text { of the lamina. }\end{array}$ & $\begin{array}{l}\text { Center on the spino-laminar junction and on the inferior } \\
\text { edge of the lamina. }\end{array}$ \\
\hline Bone resection & $\begin{array}{l}\text { Drill off the caudad half of the lamina to access the cranial } \\
\text { insertion of the YL. }\end{array}$ & $\begin{array}{l}\text { Drill off the basal half of the SP to access the cranial inser- } \\
\text { tion of the } Y L \text { and to view both medial leaves of the } Y L \text { and } \\
\text { the epidural fat in between. }\end{array}$ \\
\hline YL resection & $\begin{array}{l}\text { Ipsilateral until the lateral border of the thecal sac and de- } \\
\text { compression of the root in the lateral recess }\end{array}$ & $\begin{array}{l}\text { Tilt the table } 15^{\circ} \text { away and start contralateral decompres- } \\
\text { sion. }\end{array}$ \\
\hline Contralateral decompression & $\begin{array}{l}\text { Tilt the table } 15^{\circ} \text { away, medialize the retractor and start } \\
\text { contralateral decompression. }\end{array}$ & $\begin{array}{l}\text { Switch to the opposite side. Tilt table and retractor to get an } \\
\text { optimal line of sight between tip of the SP and medial part } \\
\text { of the facet joint. Undercut the facet preferably with } \\
\text { thin-footplate, curved } 2 \text { \& } 3 \mathrm{~mm} \text { punches. }\end{array}$ \\
\hline Final check and closure & $\begin{array}{l}\text { On both sides the lateral border of the thecal sac should pul- } \\
\text { sate freely. The traversing root should be visible up to the } \\
\text { medial wall of the pedicle. }\end{array}$ & The same \\
\hline
\end{tabular}

YL: yellow ligament, SP: spinous process. 

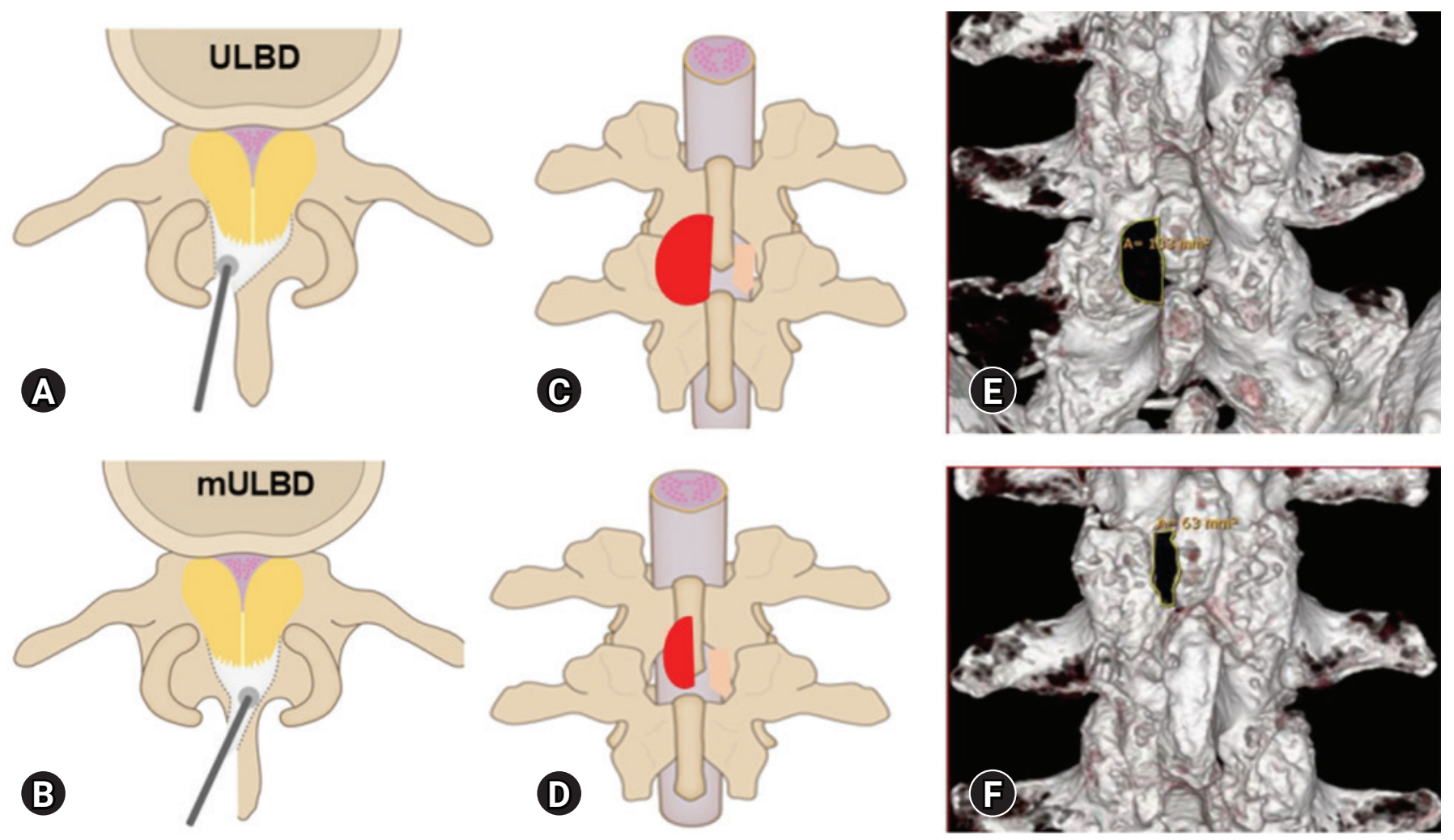

Figure 1. (A, B): conventional drilling at the lamino-facet junction on the approach side (ULBD) vs. drilling off the basal half of the spinous process at the spino-laminar junction (mULBD); $(C, D)$ area of resected bone (red); $(E, F)$ : postop CT comparing the bone defects (ULBD $133 \mathrm{~mm}^{2}$ vs. mULBD $63 \mathrm{~mm}^{2}$ ).
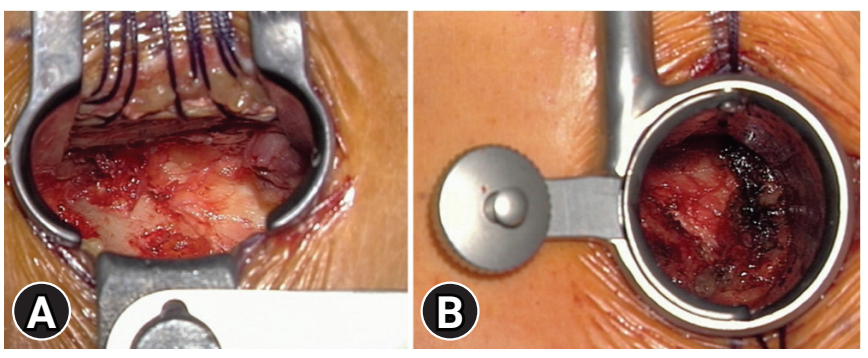

Figure 2. (A) Miniaturized speculum-retractor; (B) Tubular retractor $(\phi 18 \mathrm{~mm})$.

line epidural fat and the medial part of the contralateral YL can be seen.

Differently from the ULBD technique, as first step the contralateral YL is resected piece by piece with thin-footplate punches. The curved variant enables to slip underneath the facet and to bite the YL (Figure 3A-C). Once the contralateral dural sac and the traversing root are decompressed, the surgeon switches to the opposite side. The surgeon tilts the table back until the line of sight between SP and medial FJ is optimal. Similarly, to the previous decompression, the thickened $\mathrm{YL}$ is resected paying attention to avoid a dural tear (Figure 3D).
Meticulous epidural hemostasis is performed, and oozing bone surfaces are waxed. No drain is usually needed. Closure by layers.

Going into detail of the YL - resection, there are two options:

- To keep the YL intact as long as the bone resection (e.g. the medial part of the ipsilateral facet joint or the deep half of the contralateral lamina) is completed and then remove the YL more or less en bloc. Advantages: "safe feeling" while working with drill or rongeurs and "less pressure" when dissecting the YL from the dura. Disadvantage: unnecessary removal of bone as often the resection of the extremely thickened YL decompresses adequately the dural sac (Figure 4).

- Piecemeal resection of the YL. Advantages: mostly, no bone resection at all is necessary and a targeted decompression can be "tailored" precisely due to the early visual control of the neurostructures. Disadvantages: increased risk to tear a sticky dura and therefore time consuming repeated gently dissection of the interface between YL and dura.

In this setting we opted for the piecemeal resection as our 

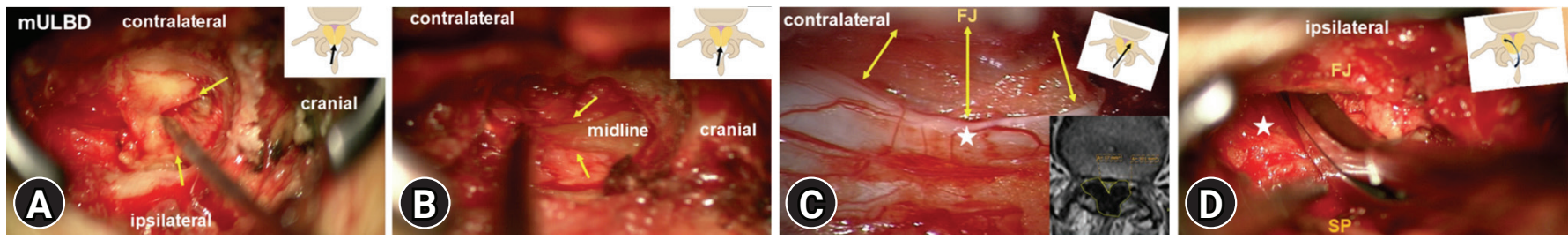

Figure 3. Intraoperative steps of mULBD: (A) The cranial insertion of the YL has been exposed. A hook separates both medial leaves (yellow arrows); (B): The epidural midline fat marks the anatomical midline between the leaves of the YL (yellow arrows). Note the symmetry of the exposure which is due to the resection of the basal half of the SP; (C) The decompression starts contralateral after tilting the table $15^{\circ}$ away from the surgeon and medializing the micro. The dura (white star) is deflected by a very thick YL (yellow arrows). In the axial MRI the YLCSA is $301 \mathrm{~mm}^{2}$ compared with merely $17 \mathrm{~mm}^{2}$ DCSA. The resection of YL decompresses the dura adequately without any additional bone drilling; (D) After switching to the contralateral side, the surgeon optimizes the line of sight between SP and medial FJ and decompresses the dura (white star). Rongeurs with curved shaft and thin footplate facilitate the resection of YL underneath the FJ.
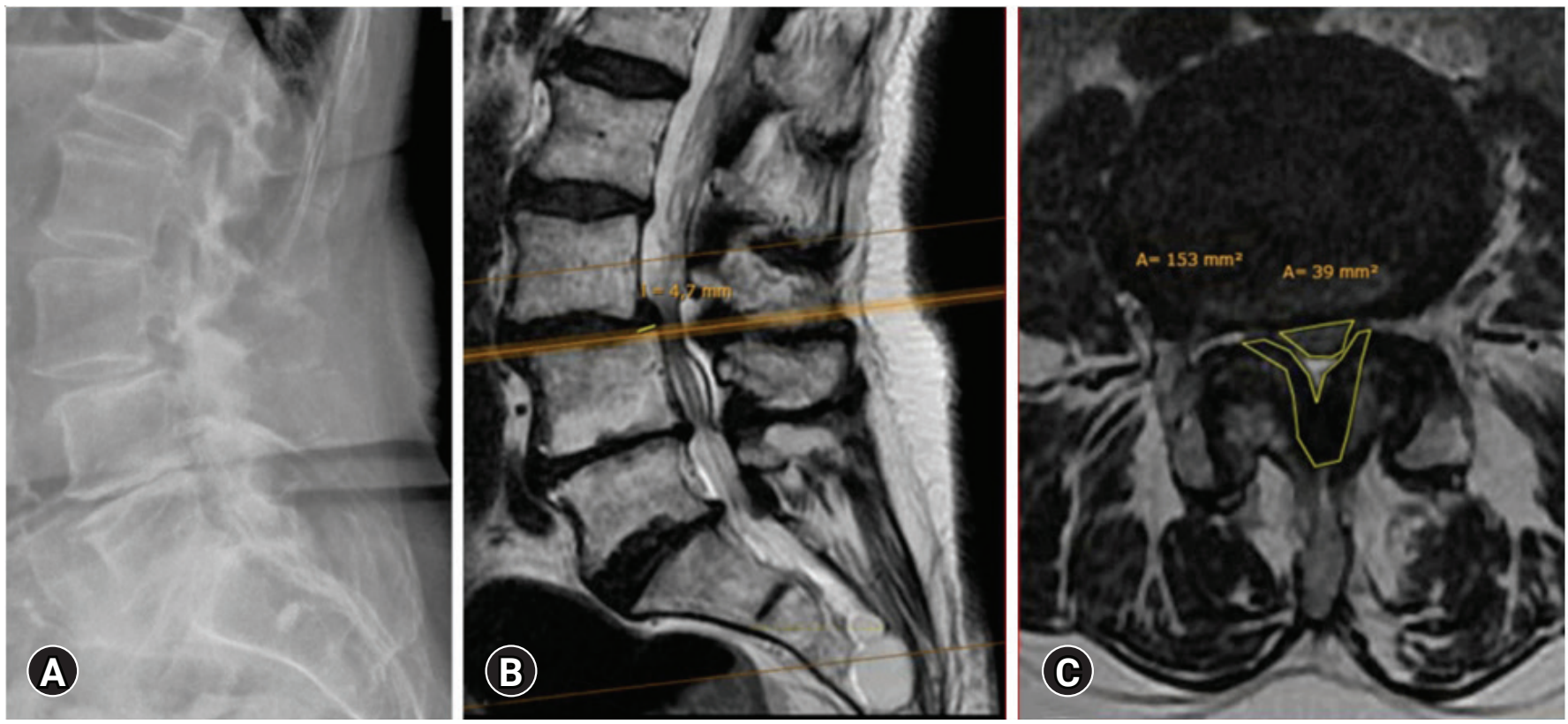

Figure 4. Clinical case: A 73-year-old lady complains about a walking distance reduced to $300 \mathrm{~m}$ due to pain (NRS 9/10) irradiating into the legs. Stooping and sitting provide relief. (A) The standing X-Ray shows a fixed slip L3/L4; (B) The MRI confirms a stenotic level L3/L4

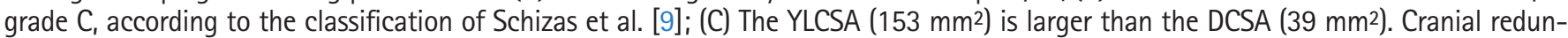
dant nerve roots are evident. The finding L4/L5 is less impressive, however requires decompression.

priority was the preservation of the maximum FJ-volume compatible with a thorough decompression.

\section{Evaluation}

\section{1) Clinical Outcomes}

Due to the restrictions imposed by the Covid-pandemia, the patients could not be followed up clinically and radiologically. They were interviewed by phone and answered the six questions of the Spinal Stenosis Measure (SSM, also known as Zuerich Claudication Questionnaire, ZCQ) [6] referring to postoperative patient's satisfaction (Likert scale responses: 1. very satisfied; 2. somewhat satisfied; 3. somewhat dissatisfied, and 4. very dissatisfied) with the overall result, the relief of pain, the ability to walk, the ability to do the housework or the job, the strength in the legs, and the balance on the feet. A score of 2.5 can be considered the cutoff between satisfied and unsatisfied patients. SSM is a disease-specific self-report outcome instrument commonly used in patients with LSS and is recommended by the North American Spine Society as the "gold standard".

\section{2) Radiological Evaluation}

A biplanar standing X-Ray was routinely performed preoperatively. Most patients presented with lumbar MRI. Due to obe- 
sity or claustrophobia, nine patients presented with lumbar CT. All patients, except two, agreed (informed consent) to undergo an ultra-low-dose -CT scan (ULD-CT; Somatom Definition AS 64, manufacturer: Siemens-Healthcare $\mathrm{GmbH}$, Erlangen, Germany) of the target facet joints pre- and post- (if preop MRI) or only postoperatively (if preop CT). Iterative reconstruction which allows for improved image quality, decreased noise (31\%), and a subsequent dose reduction (up to 60\%) was integrated into the ULD protocol [7]. Two investigators tracked independently on the bony window axial CT-slices the contours of the facet joints ( $14 \pm 3$ slices per facet joint). A specific software (Syngo.via; manufacturer: Siemens

Healthcare GmbH, Erlangen, Germany) reconstructed the volume of the facet joint (VOI, volume of interest). The reliability of the measurements with one week interval had been tested previously. The ICC results were interpreted according to Koo and $\mathrm{Li}$ [8]. The data of the first read showed "good" to "excellent" inter-rater reliability values (range: $к 0.76$ tо к 0.92 ). Inter-rater reliability for the second read ranged from "moderate" to "excellent" (range: к 0.65 tо к 0.97 ). Intra-rater reliability ranged from "good" to "excellent" for both readers (range: к 0.86 tо к 0.94 and к 0.93 to к 0.97 , respectively).

\section{3) Statistical Analysis}

Mean and standard deviation (SD) were used to describe the changes between preoperative and postoperative continuous variables, with discontinuous variables as percentages. The Levene-test was used to assess the normal distribution of values. Unpaired Student t-test was peformed for two parametric independent means. A Mann-Whitney U-test was used to compare the parameters without normal distribution. Categorical data were compared with the Fishers's exact test. $\mathrm{p}<0.05$ (two-tailed) was considered significant. Inter- and Intra-rater reliability were calculated with the use of intraclass correlation coefficients (ICC). A two-way mixed effects model with an absolute agreement definition was used [8]. Inter-rater reliability was calculated for each variable for the 1st and 2nd reads. Intra-rater reliability was calculated for each rater. The SPSS version 24.0 (SPSS Inc., Chicago, IL, USA) was used.

\section{4) Ethics Approval}

The Ethics Committee of the Federal State of Hamburg, Germany, deliberated (WF 198/20) that retrospective observational studies do not require approval whenever the data are acquired, treated and saved anonymously. This applies to the present study. The study was performed in accordance with the ethical standards as laid down in the 1964 Declaration of
Helsinki and its later amendments or comparable ethical standards.

\section{RESULTS}

\section{Clinical Outcome}

\section{1) Demographic Data}

Between 2019 and 2020, 64 Patients presenting with neurogenic claudication underwent single or multilevel decompression with ULBD (29), mULBD (29) or hybrid (6) specific for each level according to the judgement of the surgeon. By chance the cohort included 32 males and 32 females; the mean age was $81 \pm 3$ years. Forty-three pairs of facet joints were addressed with ULBD and 43 pairs with mULBD. Neither before surgery was there a significant difference between the groups in the Body Mass Index (BMI) or in symptoms as low back and leg pain and walking distance, nor after surgery in satisfaction with the surgical treatment. Demographic and clinical data, and the outcome parameters are summarized in Table 2.

\section{2) Surgical Aspects}

The parameters increasing the risk of potential postoperative segmental instability influenced the choice of the surgical technique. In the levels decompressed with mULBD the prevalence of bilateral facet joint fluid, segmental scoliotic angle, sagittal slip, low interfacettal distance and steep sagittal facet joint angle was significantly higher than in the ULBD cohort (Table 3). The latter two parameters are anatomically typical for the levels L2/ L3 and L3/L4 and become more severe in LSS. That explains why those levels accounted for almost two-third of the mULBD decompressions but for less than half of the ULBD procedures. The surgical time per level (mULBD: $70 \mathrm{~min}$ vs. ULBD $66 \mathrm{~min}$ ) was similar (Table 4). Apparently, the modifications of the mULBD were intuitive and shortened the learning curve, independently from the experience of the surgeon.

None of the patients interviewed by phone underwent revision surgery after discharge. However, three early revisions occurred: two for epidural hematoma. In one patient presenting with neurogenic claudication, L2/L3 has been treated with mULBD technique and L3/L4 with ULBD decompression. An additional fixed spondylolisthesis L4/L5 had to be decompreseed and fixed one week after the first surgery because of a persistent radicular pain L5. The further course was uneventful. Five dural lesions (ULBD: 3 vs. mULBD: 2 ) were closed intraoperatively and did not influnce the postoperative healing process. 
Table 2. Demographic and clinical data

\begin{tabular}{lccc}
\hline & mULBD & ULBD & Hybrid \\
\hline Demographics & & & \\
$\quad$ No of patients & 29 & 29 & 6 \\
Mean age ( \pm SD) & $80 \pm 3$ & $81 \pm 4$ & $80 \pm 3$ \\
Female:male & $18: 12$ & $12: 17$ & $3: 3$ \\
BMI & $26.2 \pm 3.5$ & $26.1 \pm 3.5$ & $27.2 \pm 3.3$ \\
Preoperative & & & \\
LBP (VAS) & $3.5 \pm 2.1$ & $3.1 \pm 2.7$ & $3.0 \pm 0.7$ \\
Leg pain (VAS) & $5.9 \pm 2.0$ & $6.9 \pm 1.8$ & $7.2 \pm 1.9$ \\
Walking time (min) & $10.7 \pm 9.1$ & $7.8 \pm 5.6$ & $12.6 \pm 7.3$ \\
Postoperative & & & \\
SSM-Satisfaction subscale & $12.1 \pm 5.3$ & $12.9 \pm 4.9$ & $11.3 \pm 4.4$ \\
Cut off satisfied <2.5 > unsatisfied & 2.0 & 2.1 & 1.9 \\
FU (mean; months) & 10.7 & 8.3 & 6.3 \\
Drop-out & 3 & 4 & 0 \\
\hline
\end{tabular}

BMI: body mass index, LBP: low back pain, VAS: visual analogue scale, SSM: spinal stenosis measure, SSM-Satisfaction subscale: 6 items asked (Likert score 1 to 4); total score 6 (best) to 24 (worst), Cut off: for each item, FU: follow up.

None of the difference between the parameters was statistically significant.

Table 3. Imaging data

\begin{tabular}{lcc}
\hline & mULBD & ULBD \\
\hline Stenosis acc. to Schizas et al. [9] & & \\
$\quad$ Grade B & 0 & $2(5 \%)$ \\
Grade C & $32(74 \%)$ & $25(58 \%)$ \\
Grade D & $11(26 \%)$ & $16(37 \%)$ \\
No FJ fluid (0) & $20(47 \%)$ & $28(65 \%)^{* *}$ \\
Unilateral FJ fluid (1) & $9(20 \%)$ & $8(19 \%)$ \\
Bilateral FJ fluid (2) & $14(33 \%)$ & $7(16 \%)$ \\
Patients with MRI/Xray-slip & $9(28 \%)$ & $4(13 \%)^{*}$ \\
$\quad$ mm slip (mean) & 5 & 4 \\
Scoliotic levels & $32(74 \%)$ & $23(53 \%)^{*}$ \\
$\quad$ Scoliosis & $14^{\circ} \pm 8^{\circ}$ & $12^{\circ} \pm 5^{\circ}$ \\
Disc height (mm) & $8.0 \pm 2.7$ & $9.6 \pm 2.7^{*}$ \\
Sagittal FJ angle & $25.6^{\circ} \pm 8.2^{\circ}$ & $30.2^{\circ} \pm 8.9^{\circ * *}$ \\
\hline
\end{tabular}

FJ: facet joint.

${ }^{*} \mathrm{p}<0.05 ;{ }^{* *} \mathrm{p}<0.001$; values: mean \pm SD.

\section{Radiological Evaluation}

The most relevant imaging data are shown in Table 3 [9].

The comparison of the prevalence of risk factors for potential postoperative segmental instability, which were agreed upon in the consensus meeting, showed a higher prevalence in the mULBD group. This held true for facet joint fuid, for scoliotic segmental deformity , and for marked sagittal facet angle. A hypothetical explanation for the lower disc height in the mULBD group was the more severe spine degeneration demonstrated
Table 4. Surgical data

\begin{tabular}{lcc}
\hline & mULBD & ULBD \\
\hline Level of surgery (\%) & L1/L2 (2\%) & L1/L2 (2\%) \\
& L2/L3 (30\%) & L2/L3 (14\%) \\
& L3/L4 (33\%) & L3/L4 (33\%) \\
& L4/L5 (35\%) & L4/L5 (51\%) \\
Surgical time per level (min) & $70 \pm 14$ & $66 \pm 15$ \\
DCSA preop (mm²) & $62.4 \pm 23.4$ & $50.5 \pm 17.7^{*}$ \\
DCSA postop (mm²) & $152.7 \pm 25.9$ & $151.7 \pm 30.0$ \\
FJ volume preserved (\%) & & \\
$\quad$ on the approach side & $88.42 \pm 5.58$ & $70.19 \pm 4.49^{* *}$ \\
$\quad$ on the contralateral side & $90.69 \pm 5.96$ & $86.92 \pm 6.46$
\end{tabular}

DCSA: dural cross sectional area, FJ: facet joint.

${ }^{*} \mathrm{p}<0.05 ;{ }^{* *} \mathrm{p}<0.001$; values: mean \pm SD.

by the higher prevalence of FJ-fluid and scoliotic deformity. The data confirmed the homogenous decision making process of the surgeons.

The preoperative DCSA was smaller in the ULBD cohort (51 $\mathrm{mm}^{2}$ vs. $62 \mathrm{~mm}^{2}$ ) due to higher prevalence of stenotic levels grade D (16 vs. 11) acc. to the Schizas classification. However, the postoperative DCSA was similar (ULBD: $152 \mathrm{~mm}^{2}$ vs. mULBD: $153 \mathrm{~mm}^{2}$ ). These data confirmed the non inferiority of the mULBD compared with the benchmark ULBD.

The preservation of the FJ-volume was similar in all the "oblique" approaches to the medial part of the FJ (ULBD contralateral: $88 \% \pm 6 \%$; mULBD approach side: $87 \% \pm 6 \%$; mULBD contralateral: $91 \% \pm 6 \%$ ) but significantly better than in the ipsilateral approach side of the conventional ULBD $(70 \% \pm 5 \%$; $\mathrm{p}<0.001$ ) (Figure 5). These data confirmed that mULBD when compared with ULBD provided an effective decompression of the dural sac while preserving the ipsilateral FJ-volume.

\section{DISCUSSION}

Although microsurgical decompression techniques for LSS have become very popular because they achieve satisfactory clinical and radiological results [10-12], several authors have reported the need of preserving the facet joints as much as possible [13-15]. In the daily practice three groups of patients presenting with LSS are seen: 1. Patients with stable stenotic level(s) of a lumbar spine with age-corresponding profile; 2. Patients with frankly unstable stenotic level(s); 3 . Patients with stenotic level(s) bearing risk factors, listed above, which potentially favor postoperative instability. Whereas the surgical decision making is quite straightforward in the first two groups, it may become difficult in the last one, especially if the patient 

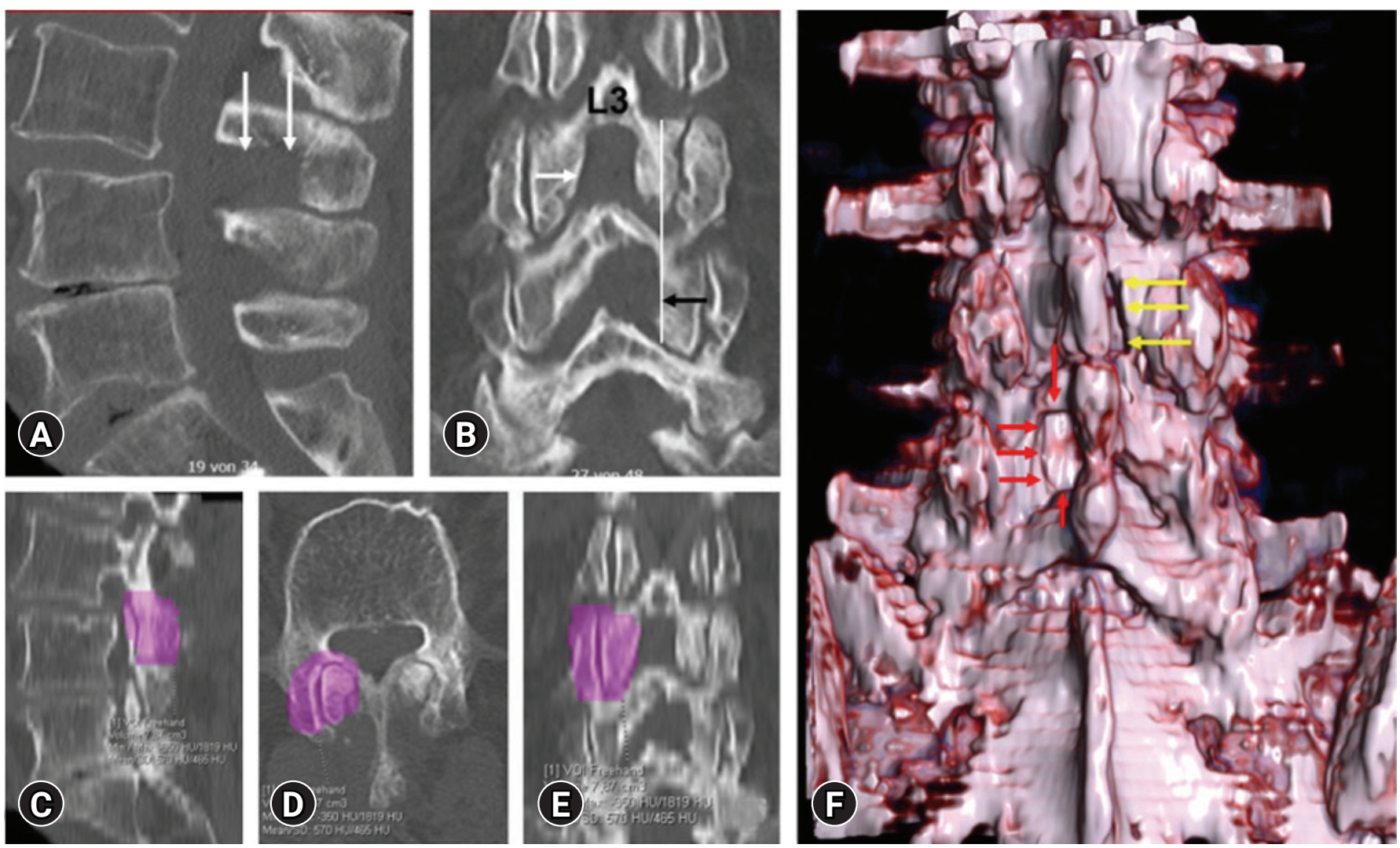

Figure 5. Postoperative ULD-CT of the same patient. (A) The basal half of the SP L3 has been drilled off; (B) On the coronal slice the L3/ L4 left-sided mULBD decompression (white arrow) is centered compared with the right-sided L4/L5 ULBD decompression (black arrow). A ULBD decompression L3/L4 would have involved more FJ (white line); (C-E) The volume of the right FJ L3/L4 measured with Syngo.via; (F) 3D-CT shows the postoperative bone defects mULBD (yellow arrows) and ULBD (red arrows).

is very reluctant toward additional fixation. The attempt to preserve completely the supra- and interspinous ligaments and the FJ as much as compatible with a thorough decompression of the dural sac via a unilateral approach has led us to melt surgical steps borrowed from ULBD and MILD.

Arai et al. [16] compared both techniques in a prospective study. The clinical improvement of the patients at two years was comparable. However, the significant more back pain following ULBD multi-level decompressions at the cranial level, $\mathrm{L} 2-\mathrm{L} 4$, was thought to be correlated with the lateral wedging on the side of the weakened facet joint shown by the AP-view of the standing X-ray. Hartmann et al. [17] had shown in a cadaver study, that detaching the supraspinous ligament caused a significant increase in ROM during flexion/extension [17]. The postoperative lower back pain following MILD was thought to be correlated with the significant higher sagittal translation at the L4/L5 level.

The learning curve of mULBD is steep, the technique does not require additional sophisticated instruments, and the rate of complications does not execeed that of comparable mini- mally invasive procedures. Alternative options are a bilateral tubular approach $[12,15]$ or an endoscopic decompression [18-20].

In several biomechanical studies the critical threshold for beginning segmental instability, usually for its rotational component, is reported to occur at $30 \%$ resection of the FJ [21-24]. Ahuja et al. [21] assessed in a finite element model study the effect of graded FJ resection. Although, compared with the control the modest increase ( $5 \%$ to $8 \%$ ) of spinal fluctuation in the sagittal and coronal plane was similar in uni- vs. bilateral $30 \%$ facetectomy, the facet loading during flexion/extension and the rise of intradiscal pressure were higher in the uni- than in the bilateral facetectomy. An explanation could be the uneven distribution of the biomechanical load. In the clinical pratice concomitant potentially destabilizing factors as low grade degenerative spondylolisthesis, sagittal oriented facet joints, scoliotic deformity, large disc height, and obesity are expected to lower the safety range. Even in anatomical normal but challenging cranial lumbar levels, with a low interfacettal distance and facet angles usually less than 35 degrees, the facet integrity 
is at risk [25].Therefore, provided a satisfactory decompression of the neural structures, the FJ can not be preserved enough.

In most patients presenting with LSS, the preoperative axial T2W MRI-slices shows at the stenotic level(s) a much larger cross-sectional area of the YL than of the dural sac. Starting the decompression by resecting the YL provides the chance of achieving an excellent relief of the dural sac while avoiding additional drilling off of biomechanical relevant bony structures. After a short learning curve this surgical option, which by the time became our favorite one, is as safe and quick as the alternative of keeping the thickend YL intact like a protection layer while enlarging the bony spinal canal.

One strength of the present study is that the volumes of the FJs have been measured with a commercially available software, originally developed for calculating tumor volumes. This method provides a better accuracy than measuring the FJs in areas or distances which do not mirror the 3D structure.

Nevertheless, limitations constrain this study. First, it is a retrospective study with the known potential bias. Second, due to the limitations caused by the pandemia the quantity and quality of the follow up data are modest. However, the clinical non-inferiority benchmark, compared with ULBD, has been reached and the radiological benchmark has been surpassed.

\section{CONCLUSION}

The medialized ULBD technique provides an effective decompression of lumbar spinal stenosis and a significant better preservation of the facet joint on the approach side than the conventional ULBD.

\section{CONFLICT OF INTEREST}

No potential conflict of interest relevant to this article.

\section{ACKNOWLEDGEMENTS}

The authors acknowledge the radiological support of Sven Opitz, MD and the clinical support of Janina Isabell Bergmann, MD, Christina Gibbert, MD, Jana Wilke; and Ralph Kothe, MD, $\mathrm{PhD}$. The former patient Dieter Witt has sponsored the english editing of the manuscript.

\section{REFERENCES}

1. Spetzger U, Bertalanffy H, Reinges MH, Gilsbach JM. Unilateral laminotomy for bilateral decompression of lumbar spinal stenosis. Part II: clinical experiences. Acta Neurochir (Wien) 1997;139:397-403.

2. Young S, Veerapen R, O'Laoire SA. Relief of lumbar canal stenosis using multilevel subarticular fenestrations as an alternative to wide laminectomy: preliminary report. Neurosurgery 1988;23:628-633.

3. Mayer HM, Heider F. "Slalom": microsurgical cross-over decompression for multilevel degenerative lumbar stenosis. Biomed Res Int 2016;2016:9074257.

4. Fujiwara Y, Manabe H, Sumida T, Izumi B, Nakanishi K, Tanaka N, et al. Facet preserving technique by en bloc flavectomy in microscopic posterior decompression surgery for lumbar spinal stenosis. semicircumferential decompression (SCD). Clin Spine Surg 2017;30:197-203.

5. Hatta Y, Shiraishi T, Sakamoto A, Yato Y, Harada T, Mikami Y, et al. Muscle-preserving interlaminar decompression for the lumbar spine: a minimally invasive new procedure for lumbar spinal canal stenosis. Spine (Phila Pa 1976) 2009;34:E276E280.

6. Stucki G, Daltroy L, Liang MH, Lipson SJ, Fossel AH, Katz JN. Measurement properties of a self-administered outcome measure in lumbar spinal stenosis. Spine (Phila Pa 1976) 1996;21:796-803.

7. Greffier J, Fernandez A, Macri F, Freitag C, Metge L, Beregi JP. Which dose for what image? Iterative reconstruction for CT scan. Diagn Interv Imaging 2013;94:1117-1121.

8. Koo TK, Li MY. A guideline of selecting and reporting intraclass correlation coefficients for reliability research. J Chiropr Med 2016;15:155-163.

9. Schizas C, Theumann N, Burn A, Tansey R, Wardlaw D, Smith FW, et al. Qualitative grading of severity of lumbar spinal stenosis based on the morphology of the dural sac on magnetic resonance images. Spine (Phila Pa 1976) 2010;35:1919-1924.

10. Oertel MF, Ryang YM, Korinth MC, Gilsbach JM, Rohde V. Long-term results of microsurgical treatment of lumbar spinal stenosis by unilateral laminotomy for bilateral decompression. Neurosurgery 2006;59:1264-1269; discussion 1269.

11. Park WB, Hong JT, Lee SW, Sung JH, Yang SH, Kim IS. Clinical and radiological comparison between ipsilateral and contralateral side canal decompression using an unilateral laminotomy approach. Korean J Spine 2016;13:41-46.

12. Schöller K, Steingrüber T, Stein M, Vogt N, Müller T, Pons-Kühnemann J, et al. Microsurgical unilateral laminotomy for decompression of lumbar spinal stenosis. longterm results and predictive factors. Acta Neurochir (Wien) 2016;158:1103-1113.

13. Klingler JH, Hubbe U, Scholz C, Krüger MT. Facet-sparing 
decompression of lumbar spinal stenosis: the minimally invasive bilateral crossover approach. J Neurol Surg A Cent Eur Neurosurg 2021;82:278-284.

14. Reinshagen C, Ruess D, Molcanyi M, Redjal N, Walcott BP, Goldbrunner R, et al. A novel translaminar crossover approach for pathologies in the lumbar hidden zone. J Clin Neurosci 2015;22:1030-1035.

15. Shin MH, Kim JS, Ryu KS, Hur JW. Bilateral decompression via microscopic tubularcrossing laminotomy (MTCL) for lumbar spinal stenosis: technique and early surgical result. Neurol Med Chir (Tokyo) 2015;55:570-577.

16. Arai Y, Hirai T, Yoshii T, Sakai K, Kato T, Enomoto M, et al. A prospective comparative study of 2 minimally invasive decompression procedures for lumbar spinal canal stenosis: unilateral laminotomy for bilateral decompression (ULBD) versus muscle-preserving interlaminar decompression (MILD). Spine (Phila Pa 1976) 2014;39:332-340.

17. Hartmann F, Janssen C, Böhm S, Hely H, Rommens PM, Gercek E. Biomechanical effect of graded minimal-invasive decompression procedures on lumbar spinal stability. Arch Orthop Trauma Surg 2012;132:1233-1239.

18. Kim HS, Wu PH, Jang IT. Lumbar endoscopic unilateral laminotomy for bilateral decompression outside-in approach: a proctorship guideline with 12 steps of effectiveness and safety. Neurospine 2020;17:S99-S109.

19. Komp M, Hahn P, Merk H, Godolias G, Ruetten S. Bilateral operation of lumbar degenerative central spinal stenosis in full-endoscopic interlaminar technique with unilateral approach: prospective 2-year results of 74 patients. J Spinal Disord Tech 2011;24:281-287.

20. Lim KT, Meceda EJA, Park CK. Inside-out approach of lumbar endoscopic unilateral laminotomy for bilateral decompression: a detailed technical description, rationale and outcomes. Neurospine 2020;17:S88-S98.

21. Ahuja S, Moideen AN, Dudhniwala AG, Karatsis E, Papadakis L, Varitis E. Lumbar stability following graded unilateral and bilateral facetectomy: a finite element model study. Clin Biomech 2020;75:105011.

22. Bresnahan L, Ogden AT, Natarajan RN, Fessler RGs. A biomechanical evaluation of graded posterior element removal for treatment of lumbar stenosis: comparison of a minimally invasive approach with two standard laminectomy techniques. Spine (Phila Pa 1976) 2009;34:17-23.

23. Hamasaki T, Tanaka N, Kim J, Okada M, Ochi M, Hutton WC. Biomechanical assessment of minimally invasive decompression for lumbar spinal canal stenosis: a cadaver study. J Spinal Disord Tech 2009;22:486-491.

24. Hasegawa K, Kitahara K, Shimoda H, Hara T. Biomechanical evaluation of destabilization following minimally invasive decompression for lumbar spinal canal stenosis. J Neurosurg Spine 2013;18:504-510.

25. Celik SE, Celik S, Kara A, Ince I, Göksu K. Lumbar facet joint angle and its importance on joint violation in lumbar microdiscectomy. Neurosurgery 2008;62:168-172; discussion 172. 